\title{
Robust gene expression programs underlie recurrent cell states and phenotype switching in melanoma.
}

Jasper Wouters ${ }^{1,2, t}$, Zeynep Kalender-Atak ${ }^{1,2,+, \$}$, Liesbeth Minnoye ${ }^{1,2}$, Katina I. Spanier ${ }^{1,2}$, Maxime De Waegeneer ${ }^{1,2}$, Carmen Bravo González-Blas ${ }^{1,2}$, David Mauduit ${ }^{1,2}$, Kristofer Davie $^{1,2}$, Gert Hulselmans ${ }^{1,2}$, Ahmad Najem ${ }^{3}$, Michael Dewaele ${ }^{4,5}$, Dennis Pedri ${ }^{1,4,5,6}$, Florian Rambow $^{4,5}$, Samira Makhzami ${ }^{1,2}$, Valerie Christiaens ${ }^{1,2}$, Frederik Ceyssens ${ }^{7}$, Ghanem Ghanem $^{3}$, Jean-Christophe Marine ${ }^{4,5}$, Suresh Poovathingal ${ }^{1}$, and Stein Aerts ${ }^{1,2, *}$

1. VIB-KU Leuven Center for Brain \& Disease Research, Leuven, Belgium.

2. KU Leuven, Department of Human Genetics, Leuven, Belgium.

3. Institut Jules Bordet, Université Libre de Bruxelles, Brussels, Belgium.

4. VIB-KU Leuven Center for Cancer Biology, Leuven, Belgium

5. KU Leuven, Department of Oncology, Leuven, Belgium.

6. KU Leuven, Department of Neurosciences, Leuven, Belgium.

7. KU Leuven, ESAT-MICAS, Leuven, Belgium.

† Equal contribution

\$ Current address: Cancer Research UK Cambridge Institute, University of Cambridge, Cambridge UK.

* Corresponding author:

Stein Aerts

Department of Human Genetics

ON IV Herestraat 49 - box 602

3000 Leuven

\section{Abstract}

Melanoma cells can switch between a melanocytic and mesenchymal-like state. Scattered evidence indicates that additional, intermediate state(s) may exist. To search for such states and decipher their underlying gene regulatory network (GRN), we studied ten melanoma cultures by single-cell RNA-seq, and 26 additional cultures by bulk RNA-seq. Although each culture exhibited a unique transcriptome, we identified shared GRNs that underlie the extreme melanocytic and mesenchymal states, and the intermediate state. This intermediate state is corroborated by a distinct chromatin landscape and governed by the transcription factors SOX6, NFATC2, EGR3, ELF1 and ETV4. Single-cell migration assays confirmed its intermediate migratory phenotype. By time-series sampling of single cells after knockdown of SOX10, we unravelled the sequential and recurrent arrangement of GRNs during phenotype switching. Jointly, these analyses indicate that an intermediate state exists and is driven by a distinct and stable "mixed" GRN rather than being a symbiotic, heterogeneous mix of cells. 


\section{Main}

Melanoma is characterised by pronounced heterogeneity due to its high mutational load ${ }^{1}$ and elevated cellular plasticity ${ }^{2}$. The latter process, called phenotype switching ${ }^{3}$, involves reversible transcriptional changes emerging from the underlying epigenome ${ }^{4}$. Overall, two main transcriptional states have been observed across cohorts of melanoma cultures ${ }^{5}$ and patient biopsies ${ }^{4,6-8}$. The melanocytic state displays high levels of lineage-specific transcription factors (TFs), including SOX10 and MITF, and functional pathways associated with the lineage, such as pigmentation. The mesenchymal-like state, also referred to as undifferentiated state, shows expression of SOX9 and activity of AP-1, and has acquired increased migratory potential and resistance to therapies. Although scattered evidence shows the existence of additional intermediate state(s) (reviewed in ${ }^{9}$ ), it remains unknown whether these states represent symbiotic mixtures of the two extreme states, or a distinct and stable "mixed" gene regulatory network (GRN).

\section{Results}

\section{Melanoma cultures exhibit distinct cell states}

To study the diversity of melanoma states we performed scRNA-seq on a cohort of melanoma samples: nine patient-derived cultures ("MM lines") ${ }^{4,10}$ and the frequently-used cell line A375 (Extended Data 1a; Supplementary Table 1,2). Analogously to scRNA-seq of biopsies in other cancers ${ }^{8,11-13}$, each sample has a unique transcriptome and forms a distinct cluster after dimensionality reduction (t-SNE; Figure 1a). Nevertheless, the cultures express many common genes, such as markers for cell cycle and their melanocytic origin (Figure 1a-d; Extended Data 1c,d). All MM lines express TFAP2A (Figure 1a; Extended Data 1c), and A375 TFAP2B (Extended Data 1c), known markers of the neural crest during development ${ }^{14,15}$ and expressed in melanocytes and melanoma cells ${ }^{16,17}$. In agreement with the antagonistic roles of SOX9 and SOX10 ${ }^{4,5,18}$, the cultures can be categorised into two groups, depending on the expression of these two TFs (Figure 1a). SOX10, a lineage TF in melanocytes together with MITF ${ }^{19}$, is expressed in seven cultures (MM001, MM011, MM031, MM057, MM074, MM087 and A375). The remaining three display expression of SOX9 (MM029, MM047 and MM099; Figure 1a). SOX9 is a marker for melanoma cells that have undergone phenotype switching towards a de-differentiated, mesenchymal-like and therapyresistant cell state ${ }^{3,20}$. The differentially expressed genes indeed correspond to signatures of melanocytic versus mesenchymal-like states ${ }^{4,5,21}$; and to a signature of pigmentation (f.i. MITF and TYR) versus epithelial-to-mesenchymal transition (EMT; f.i. TGFBI and SERPINE1) (Figure 1b-d; Extended Data 1d). The SOX9-positive MM lines also show up-regulated expression of genes required for resistance to therapies ${ }^{22,23}$ (Extended Data 1d), corroborating a phenotype switch. To identify additional common sources of variation across the melanoma samples, we performed principal component analysis (PCA) as well as canonical correlation analysis (CCA) ${ }^{24}$ (Figure $1 \mathbf{e}-\mathbf{h}$ ). The first PC reflects the distinction between melanocytic and mesenchymal-like states as demonstrated by Gene set enrichment analyses (GSEA; Supplementary Figure 1a), while high PC2 reflects the immune response, adding an additional layer of heterogeneity, mainly melanocytic samples (Figure 1e,g). CCA removes even more sample-specific differences, with $\mathrm{CC} 1$ corresponding to the melanocyticmesenchymal-like axis, and CC2 to cell-cycle-translation (Figure 1f,h; Supplementary 
Figure 1b). Together, these analyses indicate the existence of at least two subtypes of melanocytic cell states, with MM074, MM087, and MM057 (referred to as intermediate) having increased mesenchymal-like (PC1) and immune-response-like (PC2) properties when compared to MM001, MM011 and MM031. These transcriptional differences are also apparent in a supervised analysis of the melanoma AXL program ${ }^{8}$ and TGFB1 signalling ${ }^{25}$ (Figure 1i). The intermediate state exhibits increased activity of immune response genes and high resemblance to immune-like melanoma cells ${ }^{26}$ and to neural crest stem cells ${ }^{26,27}$ (Figure 1i). Examples of specific genes with higher expression across intermediate samples include $F N 1$ and S100A16 (mesenchymal-like), IFITM3 and HLA-B (immune-related), and NES and MIA (neural crest stem cell) (Figure 1j). Interestingly, intermediate samples show a higher variance in PC1/CC1 loading, indicating higher intra-sample heterogeneity (Figure 1e-h), superimposed on their increase in "mean" PC1/CC1 loading. Finally, we verified these observations using Drop-seq on a biological replicate of MM057 (Supplementary Figure 2; Supplementary Table 1).

Thus, despite each culture having a strong individualised transcriptome and a degree of variation between cells, the samples can be grouped into stable melanocytic, intermediate, and mesenchymal-like states.

\section{Transcriptional state predicts migratory capacity}

Because these three states are determined by differences in mesenchymal-like gene expression, we asked whether they present different phenotypes, such as migration. To this end, we tested their migratory capacity at single-cell level, using a collagen-coated microfluidic chip $^{28}$ (Figure 2a).

The mean squared displacement, average velocity and maximal distance reached revealed a wide variety of migratory capacities within and across melanoma cultures (Figure $\mathbf{2 b}$ ). The three mesenchymal-like MM lines cover the longest distance within 24 hours. Interestingly, two of the three intermediate cultures (MM057 and MM087), also show a high degree of cell migration (Figure 2b). The third intermediate, MM074, migrated poorly despite its intermediate transcriptome and despite high expression of migration gene signatures. A gene signature predictive for migration in bladder cancer ${ }^{29}$ follows the same global trend as the empirically measured migration, with the exception of MM074 and MM087 (Figure 2c). To determine the migratory capacity at a population level, we performed scratch-wound migration assays. Analogous visualisation of bulk and single-cell migration data highlights their significant agreement (Figure 2d; Extended Data 2), with single-cell data having higher resolution information. The disparity for MM057 potentially results from its low proliferation rate (Extended Data 1b), a factor known to complicate scratch-wound measurements ${ }^{30}$, but tractable in single-cell assays. Dimensionality reduction, using UMAP ${ }^{31}$, on the cell-feature matrix indicates a continuous gradient that strongly correlates with velocity (Figure 2e). Mesenchymal-like cultures are enriched among the fastest-migrating cells, whereas melanocytic and intermediate cultures are enriched among slowest and intermediate cells, respectively (Figure 2f).

\section{Single-cell network inference reveals candidate regulators of the intermediate state}

To determine the master regulators governing each melanoma state, we applied SCENIC network inference ${ }^{32}$. SCENIC predicts the TFs, alongside their candidate target genes, jointly 
called a regulon. To account for any potential, undesirable effect of the stochastic nature of the gradient boosting step within SCENIC, we ran the full pipeline 100 times (for a detailed description, see Methods). This strategy enables the identification of the highest-confidence regulatory interactions, and a stringent refinement of the regulons. In addition to the original motif-based SCENIC, we also performed SCENIC on a database of 2,993 epigenomic tracks, yielding regulatory interactions supported by ChIP-seq data. Collectively, these results confirm the three candidate cell states (Figure 3a,b). One cluster represents the mesenchymal-like state while the remaining SOX10-positive samples are ranked from fully melanocytic to intermediate. In the 100 SCENIC runs, we find 321 (of 973) and 43 (of 263) recurrent motifand track-based regulons, respectively, with 39 of them overlapping (91\%) (Supplementary File 1). In line with previous research ${ }^{4}$, the melanocytic state is characterized by activity of SOX10, TFAP2A, MITF, IRF4, and SOX4 (Figure 3a; Extended Data 3a). While most melanocytic regulons have a discrete activity pattern, MITF shows a gradual decrease in activity (Figure 3a; Extended Data 3d). Importantly, there is a strong correlation between the activities of motif- and track-based MITF regulons (Spearman's correlation coefficient of 0.99; Extended Data 3b), with 1,039 common target genes. Reassuringly, for most TFs (regulons shown in Figure 3a), there is a strong correlation between the activity of the motif- and trackbased regulon (Extended Data 1b,c). The mesenchymal-like state displays elevated activity of JUN, SOX9, IRF1, FOSL2, ATF5 and NFIB, in line with previous research ${ }^{4}$. The intermediate state shares several regulons with the melanocytic state including SOX10, TFAP2A and MITF (Figure 3a). The extreme melanocytic state also displays activity of the HES6 regulon (for a summary about regulons for TFs of the same family, see Supplementary Note 1). The intermediate state also shares regulons with the mesenchymal-like state, such as FOSL1 and important immune modulators IRF3 and STAT1 (Figure 3a). The EMT driver ZEB1 ${ }^{33,34}$ shows increased activity in the mesenchymal-like state, and in the highly-migratory intermediate MM057. Jointly, these data demonstrate that the intermediate state combines melanocytic and mesenchymal-like regulons within the same cell.

Finally, some regulons are enriched in the intermediate state, including SOX6, NFATC2, EGR3, ELF1 and ETV4, indicating a distinct layer in the underlying GRN (Figure 3a). Interestingly, genome-wide loss-of-function screens ${ }^{35-37}$ demonstrate dependencies for SOX6, NFATC2, ELF1 and ETV4 in melanoma cell lines (Supplementary Figure 3a). In line with this, knockdown of NFATC2 and EGR3 causes a collapse of the cell cycle and of the entire melanocytic transcriptional program while mimicking the up-regulated transcriptional programs after SOX10-KD (Supplementary Figure 3b,c). In a large cohort of primary melanomas $(n=687)^{38}$, the patients with intermediate melanoma have worse survival than others (Supplementary Figure 3d). We also detected several cell-line-specific TFs, including SOX11 and TFAP2B for A375 (Figure 3a). Finally, each culture contains cells with increased activity of cell cycle regulons such as E2F1 (Figure 3a). In line with previous reports ${ }^{39}$, the observed intratumoral heterogeneity is driven by these cell cycle-related regulons (Supplementary Figure 4).

Next, we profiled the accessible chromatin landscape using ATAC-seq ${ }^{40}$. In agreement with the transcriptome data, we observe one mesenchymal-like and two melanocytic subgroups, and identify clusters of accessible regions for each of them (Figure 3c), corroborated by previous H3K27Ac data (Extended Data 4a) ${ }^{4}$. Genomic regions accessible in both melanocytic groups (cluster 1 and 2) are enriched for SOX (confirmed by two SOX10 ChIPseq datasets 41,42; Extended Data 4b), TFAP2, E-box (E-box 1 and 2) and RUNX motifs, whereas the mesenchymal-like cultures (cluster 3 and 4 ) are characterised by AP-1, 
ATF/CREB, TEAD and NFKB motifs. SOX, MEF2, NFAT and EGR motifs are enriched in the intermediate co-accessible regions (cluster 5), and TFAP2, LEF/TCF, SOX and E-box motifs (E-box 2) in the other melanocytic cultures (cluster 6). Of note, both MITF and HES6 bind Ebox motifs, making it challenging to assign the E-boxes to either of these TFs solely from ATAC-seq data (Supplementary Note 1). Similar to our observations in the scRNA-seq data, accessible regions shared between the intermediate and mesenchymal-like cultures are enriched for AP-1 motifs (cluster 8; Figure 3c), corroborated by AP-1 ChIP-seq data 43,44 (Extended Data 4b). Interestingly, AP-1 peaks, more accessible in the intermediate cultures, are observed near their marker genes, such as FN1, IRF2, NFATC2 and SOX9 (Extended Data 4c). We also use the ATAC-seq data to refine SCENIC regulons, confirming $84 \%$ of target gene predictions (Supplementary Note 2).

Jointly, these data demonstrate that the intermediate state represents a distinct and stable transcriptional state active in all cells and shares characteristics of melanocytic and mesenchymal-like states (Figure 4; Supplementary Figure 5,6).

\section{Validation of cell states in a larger cohort of cultures and biopsies}

To examine whether the intermediate state can be consistently detected in larger cohorts of samples, we performed bulk RNA-seq on 26 additional MM lines, and new batches for seven of the above lines. The observed regulatory heterogeneity (Figure $\mathbf{3 a}, \mathbf{5 a}$ ) remains valid in this extended cohort, with the SOX10-positive cultures ranking from extreme melanocytic to intermediate (Figure 5b). We also demonstrate that the intermediate state, with enriched activity of SOX6, NFATC2, EGR3, ELF1 and ETV4, corresponds to the "transitory" subtype described by Tsoi et al. ${ }^{20}$ (Supplementary Note 3). Finally, we discovered that Hoek's cohort $\mathrm{B}^{5}$ and $\mathrm{A} 375$ are characterised by SOX11 and TFAP2B activity, and represent Tsoi's neural crest-like subgroup.

Next, we investigated the existence of the intermediate cell state in melanoma biopsies. First, we analysed a scRNA-seq data set with 2,018 malignant cells isolated from 32 melanomas 8,45 . The malignant cells of each sample form separate clusters because of their unique transcriptome, in agreement with our culture-derived scRNA-seq data (Figure 5c), whereas the (normal) host cells cluster according to cell type. Most malignant cells in this cohort display SOX10 and MITF regulon activity (Figure 5c; Extended Data 5a). A subset of cells displays co-localized SOX11 and TFAP2B activity (Extended Data 5b), thereby agreeing to A375 cells, cohort $\mathrm{B}^{5}$, and the neural crest-like subset ${ }^{20}$. Few malignant cells $(160)$ reside in the extreme melanocytic state, as identified by high HES6 regulon activity. Interestingly, cells with HES6 activity also display the highest levels of MITF regulon activity (Extended Data 5c), in agreement with the MM cultures where the extreme melanocytic state (e.g., MM001) has the highest MITF regulon activity (Extended Data 3d). Interestingly, the majority of the melanoma cells from this public data set are in the intermediate cell state, showing activity of SOX6, NFATC2 or EGR3 (1,318 of 2,018 cells or 65\%; Figure 5c; Extended Data 5a). Importantly, melanomas with high activity of one of the regulons also have elevated activity of the others, similarly to our MM lines (Extended Data 6a,b). Differential gene expression analysis on cells with and without activity of the intermediate GRN (combining SOX6, NFATC2 and EGR3 regulons) demonstrates the enrichment of immune response-related programs and of EMT, and a negative enrichment for E2F targets, thereby corroborating our observations in the MM lines (Extended Data 6c). Second, we also confirmed the intermediate state in the LMC cohort of 687 primary melanomas ${ }^{46}$, as multiple samples show high activity of the intermediate 
regulons (SOX6, NFATC2, EGR3, ELF1 and ETV4). Again, high correlation is observed between the activity of all three regulons (Supplementary Figure 7). Finally, the intermediate state is also observed in ATAC-seq data of TCGA tumours ${ }^{47}$ (Supplementary Figure 8). Interestingly, regions associated with the intermediate and mesenchymal-like cultures are more generally accessible across different cancer types as observed by others ${ }^{48}$, whereas the regions linked to melanocytic cultures are highly specific for melanoma skin cancer.

\section{SOX10 perturbation leads to common state transitions}

Next, we investigated whether phenotype switching is driven by shared GRNs, or whether they are heterogeneous, and to what extent they are influenced by the baseline GRN. Therefore, we knocked-down SOX10 in the six melanocytic cultures. First, SOX10-KD resulted in varying degrees of cell death, with the lowest levels in the intermediate samples and the highest in the extreme melanocytic cultures. Melanocytic lineage markers were extensively downregulated 72 hours after SOX10-KD, and mesenchymal-like gene signatures upregulated, to a varying degree (Figure 6a). To investigate this dynamic process in higher resolution, we performed scRNA-seq at multiple time points (Extended Data 7a), after having verified efficient knockdown of SOX10 protein by western blot (Extended Data $\mathbf{7 b}$ ). Visualization of all cells simultaneously clearly indicates the trajectory that melanoma cells follow after SOX10-KD, regardless of the type of dimensionality reduction (t-SNE or Diffusion Maps; Figure 6b), enabling us to calculate a pseudotime for every cell. Using cellAlign ${ }^{49}$, we confirmed that a highly similar trajectory is inferred by trajectory inference methods, such as SCORPIUS ${ }^{50}$ and Monocle-2 ${ }^{51}$ (Extended Data 7d). Down-regulation of SOX10 and up-regulation of SOX9 accompanies this trajectory (Figure 6c), reaffirming the antagonistic roles of the two TFs. SOX10 target genes, including MITF and TYR, decrease in expression over time and in fact lag behind SOX10 expression (Figure 6d). Although the three melanoma cultures display slightly distinct dynamics, the expression changes are highly concordant, as demonstrated by dynamic time warping, using cellAlign ${ }^{49}$ (Figure 6e). Thus, the state transition after SOX10$\mathrm{KD}$ is a recurrent phenomenon across cultures, and may therefore reflect a specific regulatory program.

The magnitude of the switch is extensive, with a collapse of the cell cycle and the entire melanocytic transcriptional program (Figure 6f,g; Extended Data 8,9). An average of 6,337 genes shows decreasing expression within 72 hours after SOX10-KD (Extended Data 9a), whereas 1,369 genes exhibit up-regulated transcription. Analysis of cell cycle genes indeed indicates that SOX10-KD results in arrest in G1 (Extended Data 7c). The up-regulated genes are also largely shared between cultures (Extended Data 9). Up-regulated processes involve migration, EMT immune cell activation, and melanoma-specific gene sets such as the melanoma TNF response ${ }^{52}$, the AXL program ${ }^{8}$ and resistance to therapies ${ }^{22}$ (Figure 6f,g; Extended Data 9). Drop-seq ${ }^{53}$ on MM057 melanoma cells before and 72 hours after SOX10KD validate the consistency of the observed transcriptional changes after SOX10-KD, i.e., the disruption of cell cycle and the melanocytic program and the resulting induction of gene sets including migration, EMT and resistance to therapies (Figure 6g; Extended Data 9b).

\footnotetext{
Network inference reveals the recurrent dynamic gene regulatory changes during phenotype switching
} 
To predict dynamic regulatory changes after SOX10-KD, we applied SCENIC network inference along the trajectories' pseudotime ${ }^{32}$. In total we detected 1,072 regulons (929 motifand 143 track-based), uniformly distributed over the three cultures, with 345 (299 and 46), 362 (309 and 53) and 365 (321 and 44) regulons identified for MM074, MM087 and MM057, respectively (for all regulons, see Supplementary File 1). The main TFs are shared across all three cultures (208). These results confirm the recurrence of the state transition and allow us to focus on the regulatory changes during phenotype switching (Supplementary Figure 911). The initial event (except for MM087) is the pausing of cell cycle (Figure 7a; Extended Data 8). Subsequently, the melanocytic core program is shut down, as evidenced by loss of SOX10 and MITF regulon activity. Additional TFs that are paused at this time include CEBPZ and MYC. Almost simultaneously, there is an increase in the unfolded protein response (UPR; e.g. XBP1 and ATF4) and in AP-1 activity (e.g. JUNB, FOSB and JUN). Simultaneously, there is an induction of ATF/CREB (e.g. CREB5) and immune-related TFs IRF/STAT (e.g. IRF9 and STAT3) become activated.

To validate these observations, we analysed ATAC-seq on the same experimental conditions for two of the melanoma cultures ${ }^{54}$. Similarly, a global collapse of the melanocytic chromatin landscape is observed, as exemplified by the loss of ATAC-seq signal at SOX10-bound regions (Figure 7b) ${ }^{41}$ and melanocytic regions (Figure 7c; cluster 1 in Figure 3c). These chromatin changes occur already 24 hours after SOX10-KD and hence precede the transcriptional switch (Figure $\mathbf{7 b}$ versus Figure $6 \mathbf{e}-\mathbf{f}$ ). On the other hand there is a gradual increase in accessibility of mesenchymal-like regions (Figure 7c; cluster 2 in Figure 3c) and of AP-1-bound regions (Figure $\mathbf{7 b}$ ) ${ }^{43}$. Because of the observed collapse of both the melanocytic transcriptome and epigenome, and the detected decrease in CDK7 activity (Extended Data 10), we hypothesised that SOX10-KD could be mimicked by a general inhibition of transcription. Therefore, we treated the cultures with THZ2, a selective inhibitor of CDK7 ${ }^{55}$, and sampled cells for RNA-seq 6 and 48 hours later. Effective treatment was confirmed by verifying downregulation of known CDK7 target genes ${ }^{56}$ (Extended Data 10). Next, we compared the transcriptional changes to those after SOX10-KD. The loss in activity of the melanocytic and cell-cycle programs is indeed recapitulated (Figure 7d). Also the enhanced activity of the UPR and AP-1 programs are mirrored by CDK7 inhibition. Unlike after SOX10-KD, no increase in the immune-related IRF/STAT transcriptional program is observed. In an additional comparison, and to investigate the effect of certain microenvironmental cues, we treated these cultures with TGF $\beta$ and TNF $\alpha$ and profiled the resulting transcriptome after 72 hours. Similar to after SOX10-KD, cell-cycle and the melanocytic programs are repressed, whereas the AP-1 and immune-related IRF/STAT transcriptional programs are up-regulated (Figure 7d). UPR displays a less pronounced increased activity after TGF $\beta / T N F \alpha$.

\section{Discussion}

Applying scRNA-seq on steady-state cultures, we detected previously known melanoma states, specifically the melanocytic and mesenchymal-like (or undifferentiated) state, and confirmed their active transcriptional programs. Importantly, we corroborated the increased migratory potential (distance and speed) of mesenchymal-like cells, using single-cell migration assays. The higher resolution of single-cell compared to bulk RNA-seq enabled us to investigate heterogeneity within cultures classified as either of these two states. The predominant driver of intratumoral heterogeneity is cell cycle, corroborating a pan-cancer study ${ }^{39}$. We also detect mesenchymal-like cells within intermediate cultures. The 
mesenchymal-like cultures on the other hand represent a more homogenous population of cells. Interestingly, we show that the heterogeneity is stable over time and across replicates of the same culture, strongly suggesting a regulated rather than stochastic process, as suggested by others ${ }^{22}$.

Recent studies have discovered a gradient of transcriptomes, from fully melanocytic over intermediate and neural crest-like to mesenchymal-like, in steady-state cultures ${ }^{20}$ and during minimal residual disease ${ }^{26}$. Here, we demonstrate that the intermediate state, corresponding to the transitory subset described by Tsoi et al. ${ }^{20}$, represents a stable and distinct cell state with underlying activity of SOX6, NFATC2, EGR3, ELF1 and ETV4 GRNs (although the latter two are not specific). These TFs have previously been linked to a more aggressive/dedifferentiated phenotype in cancer and/or in melanoma specifically ${ }^{32,57-59}$, which confirms that these cultures are in-between the melanocytic and the more deleterious mesenchymal-like state. We also determine that Hoek's cohort B cell lines and A375 display enriched activity of SOX11 and TFAP2B, and represent Tsoi's neural crest-like subgroup. Importantly, while it could a priori have been conceivable that intermediate cultures (based on bulk RNA-seq) would consist of a symbiotic mixture of cells in either the melanocytic or the mesenchymal-like state ${ }^{9}$, our results now establish that these intermediate transcriptomes are largely due to a stable "mixed gene regulatory network" that produces this intermediate transcriptome in all cells, and to a much lesser extent due to heterogeneity between the cells or random switching between the states with a specific frequency. The intermediate state shares gene regulatory networks with both the melanocytic and mesenchymal-like states, recapitulating previous observations ${ }^{5,20,21}$. Activity of most melanocytic lineage transcription factors, including SOX10 and MITF, and functional activities, such as pigmentation, are retained while a set of mesenchymal-like properties (activated immune system phenotype and increased migratory potential) are acquired, with increased activity of IRF/STAT and AP-1.

Importantly, the intermediate state also occurs in melanoma biopsies as it can be detected in (single-cell) RNA- and ATAC-seq and in multiple patient cohorts. Besides their increased mesenchymal properties, we observed the immune response-like molecular phenotype (derived from our intermediate state) in melanoma biopsies. Similar cells with a 'mixed-lineage' phenotype and with elevated expression of immune and inflammation response genes were recently discovered in lung cancer ${ }^{60}$. Interestingly, these cancer cells were able to evade the NK-cell-mediated immune surveillance ${ }^{60}$. Also in pancreatic cancer, a subset of antigenpresenting ductal cells was recently detected, expressing major histocompatibility complex class II genes and complement pathway components ${ }^{61}$. And finally, gene sets related to immune responses were observed as marker genes for BRAFi-resistant melanoma cells in a functional genomics screen ${ }^{62}$. Altogether, this makes these specific transcription factors attractive candidates for targeted melanoma therapy, or cancer in general. NFATC2 and ELF1 have been suggested and assessed before as potential therapeutic targets in melanoma ${ }^{59,63}$. In addition, EGR3 inhibition has been shown to block AP-1 activity upon NGFR activation ${ }^{64}$.

Despite representing stable and distinct cell states in steady-state conditions, melanoma cells can switch to alternative states using specific microenvironmental stimuli such as TGF $\beta$ and/or TNFa. Here, we demonstrate that SOX10-KD is sufficient to switch melanocytic and intermediate to mesenchymal-like cells, and that the resulting cells acquire the same transcriptional programs for migration, invasion and resistance to therapy as mesenchymallike cells $4,22,23,29$. This observation strongly suggests that SOX10 directly or indirectly suppresses the mesenchymal-like state and the associated GRNs. Although SOX10 has been suggested as a transcriptional repressor in breast cancer ${ }^{65}$, we could not detect any evidence 
for such a role in melanoma. Alternatively, given the large-scale effects of SOX10-KD on gene expression, the phenotype switch likely results from the loss of multiple SOX10-controlled repressors, including transcriptional and post-translational repressors, such as its direct targets DUSP6, MXI1 and NFATC2 ${ }^{66-69}$. We also demonstrate that the inhibition of CDK7dependent transcription mimics the extensive effects of SOX10-KD on gene expression, leading to the inhibition of cell cycle and the melanocytic program, but also the increasing activity of the AP-1 program and the unfolded protein response. This suggests that the upregulation of the AP-1 program can at least in part be due to a stress response, resulting from the collapsing transcriptome. On the other hand, genes up-regulated after SOX10-KD include SOX9 and other markers from the mesenchymal-like melanoma state, arguing against a pure stress response signature.

In conclusion, we used single-cell transcriptomics combined with gene regulatory network and trajectory inference to map the gene regulatory landscape of recurrent melanoma cell states. We find that transcriptional and phenotypic heterogeneity can be largely attributed to differences in gene regulatory networks, supplemented with some degree of stochasticity; and that phenotype switching from the melanocytic to the mesenchymal state is controlled by a regulatory program that is highly reproducible across distinct patient cultures. 


\section{Acknowledgements}

This work was funded by an ERC Consolidator Grant to S. Aerts (no. 724226_cis-CONTROL), and by the KU Leuven (grant no. C14/18/092 to S. Aerts), the Harry J. Lloyd Charitable Trust, the Foundation Against Cancer (grant no, 2016-070 to S. Aerts), PhD fellowships from the FWO (L.M., no. 1S03317N) and a postdoctoral research fellowship from Kom op tegen Kanker (Stand up to Cancer), the Flemish Cancer Society and from Stichting tegen Kanker (Foundation against Cancer), the Belgian Cancer Society (J.W). We would like to thank Joanna Poźniak for help with the Leeds Melanoma Cohort, and Tim Stakenborgs en Evelien Mathieu (IMEC) for help with the single-cell migration and lens-free imaging setup. Computing was performed at the Vlaamse Supercomputer Center and high-throughput sequencing via the Genomics Core Leuven. Single-cell infrastructure was funded by the Hercules Foundation (grant no. AKUL/13/41). The funders had no role in study design, data collection and analysis, decision to publish or preparation of the manuscript.

The results of this publication are based partly on data generated by the University of Leeds in connection with the project 'The Leeds Melanoma Cohort'; otherwise known as Melanoma Follow-up and Case-Control Family Study (REC reference number 01/03/057). This data is currently held within the European Genome-phenome Archive at the European Bioinformatics Institute (accession number EGAS00000000029). The generation of this data was funded by Cancer Research UK (award refs C588/A19167, C8216/A6129 and C588/A10721) and with the support of the National Institutes of Health (award ref. CA83115) and the European Commission Horizon 2020 Research and Innovation Programme (Ref. No. 641458).

\section{Author Contributions}

J.W., Z.K.A. and S.A. conceived the study. J.W. performed the experimental work for the $10 \mathrm{x}$ scRNA-seq datasets with the help of V.C., S.M. and S.P.. J.W. performed the experimental work for the Drop-seq datasets with the help of K.D. and V.C.. J.W. and L.M. performed the experimental work for the Omni-ATAC datasets with the help of D.M. and V.C.. L.M. performed the experimental work and analysis of the single-cell migration assays with the help of F.C. and S.P.. D.P. performed the experimental work and analysis of the bulk migration assays with the help of J.W.. J.W., Z.K.A. and K.I.S. performed all bioinformatics analyses with the help of G.H., M.D.W., K.D. and C.B.G.B.. A.N. and G.G. provided valuable melanoma cultures. M.D., F.R. and J.C.M. provided valuable materials and contributed to the manuscript. J.W., Z.K.A., K.I.S. and S.A. wrote the manuscript.

\section{Competing Interests}

The authors declare no conflict of interest. 


\section{References}

1. Alexandrov, L. B. et al. Signatures of mutational processes in human cancer. Nature 500, 415-421 (2013).

2. Grzywa, T. M., Paskal, W. \& Włodarski, P. K. Intratumor and Intertumor Heterogeneity in Melanoma. Transl. Oncol. 10, 956-975 (2017).

3. Hoek, K. S. \& Goding, C. R. Cancer stem cells versus phenotype-switching in melanoma. Pigment Cell Melanoma Res. 23, 746-759 (2010).

4. Verfaillie, A. et al. Decoding the regulatory landscape of melanoma reveals TEADS as regulators of the invasive cell state. Nat. Commun. 6, 6683 (2015).

5. Hoek, K. S. et al. Metastatic potential of melanomas defined by specific gene expression profiles with no BRAF signature. Pigment Cell Res. 19, 290-302 (2006).

6. Hoek, K. S. et al. In vivo switching of human melanoma cells between proliferative and invasive states. Cancer Res. 68, 650-656 (2008).

7. Wouters, J. et al. A novel hypoxia-associated subset of FN1 high MITF low melanoma cells: identification, characterization, and prognostic value. Mod. Pathol. Off. J. U. S. Can. Acad. Pathol. Inc 27, 1088-1100 (2014).

8. Tirosh, I. et al. Dissecting the multicellular ecosystem of metastatic melanoma by single-cell RNA-seq. Science 352, 189-196 (2016).

9. Rambow, F., Marine, J.-C. \& Goding, C. R. Melanoma plasticity and phenotypic diversity: therapeutic barriers and opportunities. Genes Dev. 33, 1295-1318 (2019).

10. Gembarska, A. et al. MDM4 is a key therapeutic target in cutaneous melanoma. Nat. Med. 18, 1239-1247 (2012).

11. Venteicher, A. S. et al. Decoupling genetics, lineages, and microenvironment in IDHmutant gliomas by single-cell RNA-seq. Science 355, (2017).

12. Puram, S. V. et al. Single-Cell Transcriptomic Analysis of Primary and Metastatic Tumor Ecosystems in Head and Neck Cancer. Cell 171, 1611-1624.e24 (2017).

13. Lambrechts, D. et al. Phenotype molding of stromal cells in the lung tumor microenvironment. Nat. Med. 24, 1277 (2018). 
14. Sargent, T. D. Transcriptional regulation at the neural plate border. Adv. Exp. Med. Biol. 589, 32-44 (2006).

15. Van Otterloo, E., Li, H., Jones, K. L. \& Williams, T. AP-2 $\alpha$ and AP-2 $\beta$ cooperatively orchestrate homeobox gene expression during branchial arch patterning. Dev. Camb. Engl. 145, (2018).

16. Seberg, H. E. et al. TFAP2 paralogs regulate melanocyte differentiation in parallel with MITF. PLoS Genet. 13, e1006636 (2017).

17. Wouters, J. et al. Comprehensive DNA methylation study identifies novel progressionrelated and prognostic markers for cutaneous melanoma. BMC Med. 15, 101 (2017).

18. Shakhova, O. et al. Antagonistic cross-regulation between Sox9 and Sox10 controls an anti-tumorigenic program in melanoma. PLoS Genet. 11, e1004877 (2015).

19. Southard-Smith, E. M., Kos, L. \& Pavan, W. J. Sox10 mutation disrupts neural crest development in Dom Hirschsprung mouse model. Nat. Genet. 18, 60-64 (1998).

20. Tsoi, J. et al. Multi-stage Differentiation Defines Melanoma Subtypes with Differential Vulnerability to Drug-Induced Iron-Dependent Oxidative Stress. Cancer Cell 33, 890904.e5 (2018).

21. Widmer, D. S. et al. Systematic classification of melanoma cells by phenotype-specific gene expression mapping. Pigment Cell Melanoma Res. 25, 343-353 (2012).

22. Shaffer, S. M. et al. Rare cell variability and drug-induced reprogramming as a mode of cancer drug resistance. Nature 546, 431-435 (2017).

23. Hugo, W. et al. Genomic and Transcriptomic Features of Response to Anti-PD-1 Therapy in Metastatic Melanoma. Cell 165, 35-44 (2016).

24. Butler, A., Hoffman, P., Smibert, P., Papalexi, E. \& Satija, R. Integrating single-cell transcriptomic data across different conditions, technologies, and species. Nat. Biotechnol. 36, 411-420 (2018).

25. Karakas, B. et al. Interleukin-1 alpha mediates the growth proliferative effects of transforming growth factor-beta in p21 null MCF-10A human mammary epithelial cells. Oncogene 25, 5561-5569 (2006). 
26. Rambow, F. et al. Toward Minimal Residual Disease-Directed Therapy in Melanoma. Cell 174, 843-855.e19 (2018).

27. Lee, G. et al. Isolation and directed differentiation of neural crest stem cells derived from human embryonic stem cells. Nat. Biotechnol. 25, 1468-1475 (2007).

28. Mathieu, E. et al. Time-lapse lens-free imaging of cell migration in diverse physical microenvironments. Lab. Chip 16, 3304-3316 (2016).

29. Wu, Y., Siadaty, M. S., Berens, M. E., Hampton, G. M. \& Theodorescu, D. Overlapping gene expression profiles of cell migration and tumor invasion in human bladder cancer identify metallothionein $1 \mathrm{E}$ and nicotinamide $\mathrm{N}$-methyltransferase as novel regulators of cell migration. Oncogene 27, 6679-6689 (2008).

30. Kramer, N. et al. In vitro cell migration and invasion assays. Mutat. Res. $\mathbf{7 5 2}, 10-24$ (2013).

31. Becht, E. et al. Dimensionality reduction for visualizing single-cell data using UMAP. Nat. Biotechnol. (2018) doi:10.1038/nbt.4314.

32. Aibar, S. et al. SCENIC: single-cell regulatory network inference and clustering. Nat. Methods 14, 1083-1086 (2017).

33. Caramel, J. et al. A switch in the expression of embryonic EMT-inducers drives the development of malignant melanoma. Cancer Cell 24, 466-480 (2013).

34. Denecker, G. et al. Identification of a ZEB2-MITF-ZEB1 transcriptional network that controls melanogenesis and melanoma progression. Cell Death Differ. 21, 1250-1261 (2014).

35. Meyers, R. M. et al. Computational correction of copy number effect improves specificity of CRISPR-Cas9 essentiality screens in cancer cells. Nat. Genet. 49, 1779$1784(2017)$.

36. McFarland, J. M. et al. Improved estimation of cancer dependencies from large-scale RNAi screens using model-based normalization and data integration. Nat. Commun. 9, $4610(2018)$

37. Dempster, J. M. et al. Extracting Biological Insights from the Project Achilles Genome- 
Scale CRISPR Screens in Cancer Cell Lines.

http://biorxiv.org/lookup/doi/10.1101/720243 (2019) doi:10.1101/720243.

38. Nsengimana, J. et al. $\beta$-Catenin-mediated immune evasion pathway frequently operates in primary cutaneous melanomas. J. Clin. Invest. 128, 2048-2063 (2018).

39. Kinker, G. S. et al. Pan-cancer single cell RNA-seq uncovers recurring programs of cellular heterogeneity. http://biorxiv.org/lookup/doi/10.1101/807552 (2019) doi:10.1101/807552.

40. Corces, M. R. et al. An improved ATAC-seq protocol reduces background and enables interrogation of frozen tissues. Nat. Methods 14, 959-962 (2017).

41. Laurette, P. et al. Transcription factor MITF and remodeller BRG1 define chromatin organisation at regulatory elements in melanoma cells. eLife 4, (2015).

42. Eskiocak, B. et al. Biomarker Accessible and Chemically Addressable Mechanistic Subtypes of BRAF Melanoma. Cancer Discov. 7, 832-851 (2017).

43. Gertz, J. et al. Distinct properties of cell-type-specific and shared transcription factor binding sites. Mol. Cell 52, 25-36 (2013).

44. Joseph, R. et al. Integrative model of genomic factors for determining binding site selection by estrogen receptor-a. Mol. Syst. Biol. 6, 456 (2010).

45. Jerby-Arnon, L. et al. A Cancer Cell Program Promotes T Cell Exclusion and Resistance to Checkpoint Blockade. Cell 175, 984-997.e24 (2018).

46. Thakur, R. et al. Transcriptomic Analysis Reveals Prognostic Molecular Signatures of Stage I Melanoma. Clin. Cancer Res. 25, 7424-7435 (2019).

47. Corces, M. R. et al. The chromatin accessibility landscape of primary human cancers. Science 362, eaav1898 (2018).

48. Rambow, F. et al. New Functional Signatures for Understanding Melanoma Biology from Tumor Cell Lineage-Specific Analysis. Cell Rep. 13, 840-853 (2015).

49. Alpert, A., Moore, L. S., Dubovik, T. \& Shen-Orr, S. S. Alignment of single-cell trajectories to compare cellular expression dynamics. Nat. Methods 15, 267-270 (2018). 
50. Cannoodt, R. et al. SCORPIUS improves trajectory inference and identifies novel modules in dendritic cell development. bioRxiv 079509 (2016) doi:10.1101/079509.

51. Qiu, X. et al. Reversed graph embedding resolves complex single-cell trajectories. Nat. Methods 14, 979-982 (2017).

52. Riesenberg, S. et al. MITF and c-Jun antagonism interconnects melanoma dedifferentiation with pro-inflammatory cytokine responsiveness and myeloid cell recruitment. Nat. Commun. 6, 8755 (2015).

53. Macosko, E. Z. et al. Highly Parallel Genome-wide Expression Profiling of Individual Cells Using Nanoliter Droplets. Cell 161, 1202-1214 (2015).

54. Bravo González-Blas, C. et al. cisTopic: cis-regulatory topic modeling on single-cell ATAC-seq data. Nat. Methods 16, 397-400 (2019).

55. Wang, Y. et al. CDK7-dependent transcriptional addiction in triple-negative breast cancer. Cell 163, 174-186 (2015).

56. Eliades, P. et al. High MITF Expression Is Associated with Super-Enhancers and Suppressed by CDK7 Inhibition in Melanoma. J. Invest. Dermatol. 138, 1582-1590 (2018).

57. Lisbôa-Nascimento, T. et al. Identification of Melanoma Stem Cells in Long-term Cultures and of SOX6 as a Specific Biomarker for these Stem Cells. J Can Epi Treat 1, $15-27$ (2015).

58. Baron, V. T., Pio, R., Jia, Z. \& Mercola, D. Early Growth Response 3 regulates genes of inflammation and directly activates IL6 and IL8 expression in prostate cancer. Br. J. Cancer 112, 755-764 (2015).

59. Perotti, V. et al. NFATc2 is an intrinsic regulator of melanoma dedifferentiation. Oncogene 35, 2862-2872 (2016).

60. Laughney, A. M. et al. Regenerative lineages and immune-mediated pruning in lung cancer metastasis. Nat. Med. 26, 259-269 (2020).

61. Moncada, R. et al. Integrating microarray-based spatial transcriptomics and single-cell RNA-seq reveals tissue architecture in pancreatic ductal adenocarcinomas. Nat. 
Biotechnol. 38, 333-342 (2020).

62. Jun, S., Lim, H., Chun, H., Lee, J. H. \& Bang, D. Single-cell analysis of a mutant library generated using CRISPR-guided deaminase in human melanoma cells. Commun. Biol. $3,1-12(2020)$.

63. Huang, X. et al. Critical role for the Ets transcription factor ELF-1 in the development of tumor angiogenesis. Blood 107, 3153-3160 (2006).

64. Levkovitz, Y. \& Baraban, J. M. A dominant negative Egr inhibitor blocks nerve growth factor-induced neurite outgrowth by suppressing c-Jun activation: role of an Egr/c-Jun complex. J. Neurosci. Off. J. Soc. Neurosci. 22, 3845-3854 (2002).

65. Dravis, C. et al. Epigenetic and Transcriptomic Profiling of Mammary Gland Development and Tumor Models Disclose Regulators of Cell State Plasticity. Cancer Cell 34, 466-482.e6 (2018).

66. Schreiber-Agus, N. et al. An amino-terminal domain of Mxi1 mediates anti-Myc oncogenic activity and interacts with a homolog of the yeast transcriptional repressor SIN3. Cell 80, 777-786 (1995).

67. Lee, T. C. \& Ziff, E. B. Mxi1 is a repressor of the c-Myc promoter and reverses activation by USF. J. Biol. Chem. 274, 595-606 (1999).

68. Carvalho, L. D. S. et al. The NFAT1 transcription factor is a repressor of cyclin A2 gene expression. Cell Cycle Georget. Tex 6, 1789-1795 (2007).

69. Wong, V. C. L. et al. Tumor suppressor dual-specificity phosphatase 6 (DUSP6) impairs cell invasion and epithelial-mesenchymal transition (EMT)-associated phenotype. Int. J. Cancer 130, 83-95 (2012). 


\section{Figure Legends}

Figure 1: Melanoma cultures exhibit distinct cell states. Nine patient-derived MM lines and cell line A375 were profiled using scRNA-seq. a, The cells cluster according to their cell line origin in a t-SNE. All lines except A375 express the neural crest marker TFAP2A. Based on expression of SOX10 and SOX9, the cultures split into melanocytic and mesenchymal-like melanomas. b, Melanocytic and mesenchymal-like gene signatures define two groups among the cultures, with A375, MM029, MM047 and MM099 having a mesenchymal-like transcriptome. c, Melanocytic cultures show high expression of a pigmentation gene signature (GO), and melanogenesis-related genes, MITF and TYR. $\mathbf{d}$, The mesenchymal-like cultures have higher expression of a gene signature for epithelial-to-mesenchymal transition (Hallmark), and MM029, MM047 and MM099 express the epithelial-to-mesenchymaltransition genes TGFBI and SERPINE1. e-f, Both principal component (e) and canonical component (f) analysis order the cells in a gradient from melanocytic to mesenchymal-like on the first axis, as illustrated by AUCell activity. The second axis correlates with immune response (PC2) and cell cycle (CC2). $\mathbf{g}$-h, Three of the melanocytic cultures (now called 'intermediate') show higher mean and variance in PC1 loading (g), and higher variance in CC1 loading $(\mathrm{h})$, compared to the other melanocytic cultures. Box plots display the median as centre line and the upper and lower quartiles as box limits. i, The melanoma AXL program, and TBFb1 signalling, increase in expression from extreme melanocytic over intermediate to mesenchymal-like cultures. The intermediate cultures show higher expression than the melanocytic lines in immune activation genes (KEGG graft versus host disease), and gene signatures characteristic for immune- and neural crest-like melanoma cells, and for neural crest stem cells. j, The mesenchymal-like genes FN1 and S100A16, along with the immunerelated genes IFITM 3 and $H L A-B$, are highly expressed in the intermediate and mesenchymallike cultures, while the neural-crest-stem-cell markers NES and MIA are specific to the intermediate cultures. P-values were calculated using MAST (for genes) and the Wilcoxon rank-sum test with Benjamini-Hochberg FDR correction (for gene signatures), both two-sided $(n=4,322)$. Violin plots display cell densities as shape and cells as dots.

Figure 2: Transcriptional state predicts migratory capacity. a, Our single-cell migration assay set-up using lens-free imaging. Left: A microfluidic device containing single-cell migration channels is loaded with cells, covered with a glass slide and placed on top of a complementary metal-oxide-semiconductor sensor (CMOS) that detects diffraction of the emitted laser though the migration device to track the movement of single cells. Middle: Layout of the microfluidic migration device. Cells are loaded in the seeding channel and migrate through the small migration channels $(6 \times 10 \mu \mathrm{m})$. Right: Reconstructed image of cells (encircled) moving in the migration channels in the direction of the red arrows. $b$, The mean square displacement $(n=368)$, velocity $(n=368)$ and maximal distance $(n=370)$ travelled of single cells show a gradual increase from melanocytic to mesenchymal-like cultures. c, The expression of cell migratory potential genes correlates with the migratory behaviour. d, The migratory behaviour of single cells in the MM lines replicates measurements from a bulk migration assay, with the cultures classified as mesenchymal-like migrating most rapidly (error bars show SEM). e-f, A UMAP of single cells $(n=368)$ based on their migratory capabilities shows that the velocity of $(e)$ correlates with the state of the cells $(f)$. P-values were calculated using the Wilcoxon rank-sum test with Benjamini-Hochberg FDR correction, two-sided. Box plots display the median as centre line and the upper and lower quartiles as box limits. 
Figure 3: Single-cell network inference reveals candidate regulators of the intermediate state. a, The heatmap shows a selection of the regulons identified by SCENIC (rows), and their activities in each cell (columns). For selected transcription factors (TFs) the DNA-binding motif (PWM) is shown, predicted by the RcisTarget step of SCENIC. $\mathbf{b}$, A UMAP based on SCENIC regulons separates the intermediate from the other melanocytic cultures in an unsupervised manner. c, Chromatin accessibility, measured by ATAC-seq in the MM lines, establishes three clusters, corresponding to the previously described cell states, and nine clusters of genomic regions. For each cluster of regions, the enriched DNA-binding motifs and associated TFs are indicated.

Figure 4: Results of SCENIC runs on multiple data sets pruned to a high-confidence gene regulatory network. The gene expression in the (a) intermediate, (b) melanocytic and (c) mesenchymal-like MM lines is shown as node colour. The edge width corresponds to the number of SCENIC runs in which the TF-target interaction is predicted, and double lines indicate connections recovered in both motif- and track-based databases. The network consists of several subnetworks (transcriptional programs, encircled), of which some are differentially expressed between melanoma states (arrows). This network is available at NDEx: https://doi.org/10.18119/N99C71.

Figure 5: Validation of cell states in a larger cohort of cultures and biopsies. a, Violin plots showing the activity of SCENIC regulons that are enriched in the intermediate cultures, as measured by AUCell. P-values were calculated using the Wilcoxon rank-sum test with Benjamini-Hochberg FDR correction, two-sided $(n=4,322)$. Violin plots display cell densities as shape and cells as dots. b, 26 additional and 7 previous MM lines cluster into an extreme melanocytic, intermediate and mesenchymal-like cell state on the AUCell values of the previously defined regulons ( $n=33)$. Additionally, we recover a previously described 'cohort B', characterized by SOX11 and TFAP2B activity. c, The malignant cells in a pool of single cell biopsies contain melanocytic cells (high expression of SOX10 and MITF regulons) and cells in the intermediate state (activity of SOX6, NFATC2 and EGR3 regulons, as measured by AUCell; $n=2,018$ malignant cells and a total of 7,186 cells; see also Extended Data 5).

Figure 6: SOX10 perturbation leads to common state transitions. a, In the SOX10expressing MM lines, 72 hours after knockdown (KD) of SOX10, the melanocytic gene signature decreases in expression (top), while the mesenchymal-like signature increases (bottom). Semi-quantitative assessment of cell death is indicated at the bottom ('+++': high cell death; '++': intermediate; '+': low cell death). b, Seurat t-SNE (left) and Diffusion Map (DM; right) with cells coloured according to the experimental time point, indicate that melanoma cells follow a trajectory after SOX10-KD (arrows). c, While SOX10 is down-regulated along the trajectory from control to $72 \mathrm{~h}$ SOX10-KD, SOX9 is up-regulated. d, Expression of established SOX10 target genes (rows) in each cell (columns) grouped by experimental time point. The three cultures display distinct dynamics, with the switch from a melanocytic to a mesenchymal-like transcriptional program occurring before $24 \mathrm{~h}$ KD (MM087), after $24 \mathrm{~h}$ (MM057) or after $48 \mathrm{~h}$ (MM074), indicated by asterisks. e, Comparative alignment of transition trajectories by applying dynamic time warping shows the optimal alignment (white line) through 
a dissimilarity matrix. Despite the varying dynamics (asterisks indicate the switch from a melanocytic to a mesenchymal transcriptional program), the expression changes between the three cultures are highly concordant. $\mathbf{f}$, AUCell of gene signatures on the single-cell SOX10$\mathrm{KD}$ shows that melanocytic, cell cycle (hallmark) and pigmentation decrease, while mesenchymal-like, cell migration and therapy resistance related genes increase. g, The AUCell violin plots show an increase in expression of the epithelial-to-mesenchymal transition and the melanoma TNF response gene signatures upon SOX10-KD in all three melanoma cultures. The Drop-seq experiment on a biological replicate of the KD in MM057 reproduces the 10x Chromium results. Violin plots display cell densities as shape $(n=27,163)$.

Figure 7: Network inference reveals the recurrent dynamic gene regulatory changes during phenotype switching. a, The heatmaps show selected regulons that are recovered recurrently in the SOX10 knockdown (KD) experiments on the three intermediate melanoma cultures. Cells are ordered according to their diffusion maps' pseudotime (see also Figure $6 \mathrm{~b}, \mathrm{e})$. For each regulon, the transcription factor (TF), the number of predicted target genes, and the recurrence in 100 SCENIC runs are indicated ( ${ }^{*}$ indicates track-based regulon). b, The chromatin accessibility at SOX10 and JUND-bound regions (defined by ChIP-seq experiments) is affected by SOX10-KD. While SOX10-bound regions close after knockdown, JUND-bound regions show a slight increase in ATAC-seq signal. c, The melanocytic and mesenchymal-like chromatin regions identified in the nine MM lines (cluster 1 and 2 for melanocytic; cluster 3 and 4 for mesenchymal-like regions from Figure 3c) show a gradual decrease, respectively increase, in accessibility after SOX10-KD. The plots show the cumulative normalized ATAC-seq signal of the respective regions. d, The gene expression changes after SOX10-KD are mimicked by CDK7 inhibition with THZ2 and TGF $/$ TNFa treatment. While melanocytic and cell cycle-related subnetworks of the pruned melanoma network (Figure 4) are repressed in all three treatments, mesenchymal, and partially unfoldedprotein-response are up-regulated. SOX10-KD: aggregate signal of the three single-cell SOX10-KD experiments. 


\section{Figure 1}

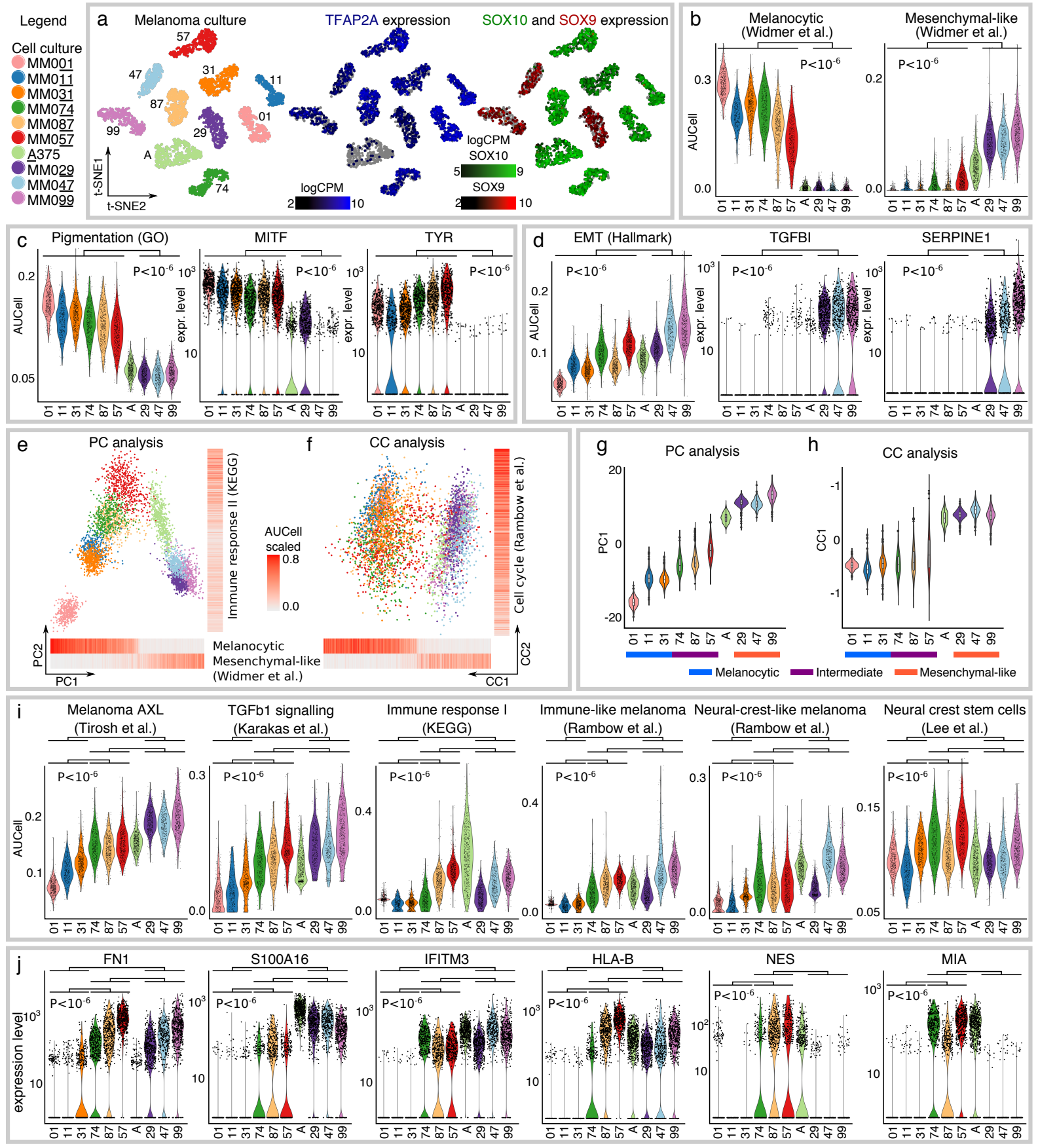


Figure 2
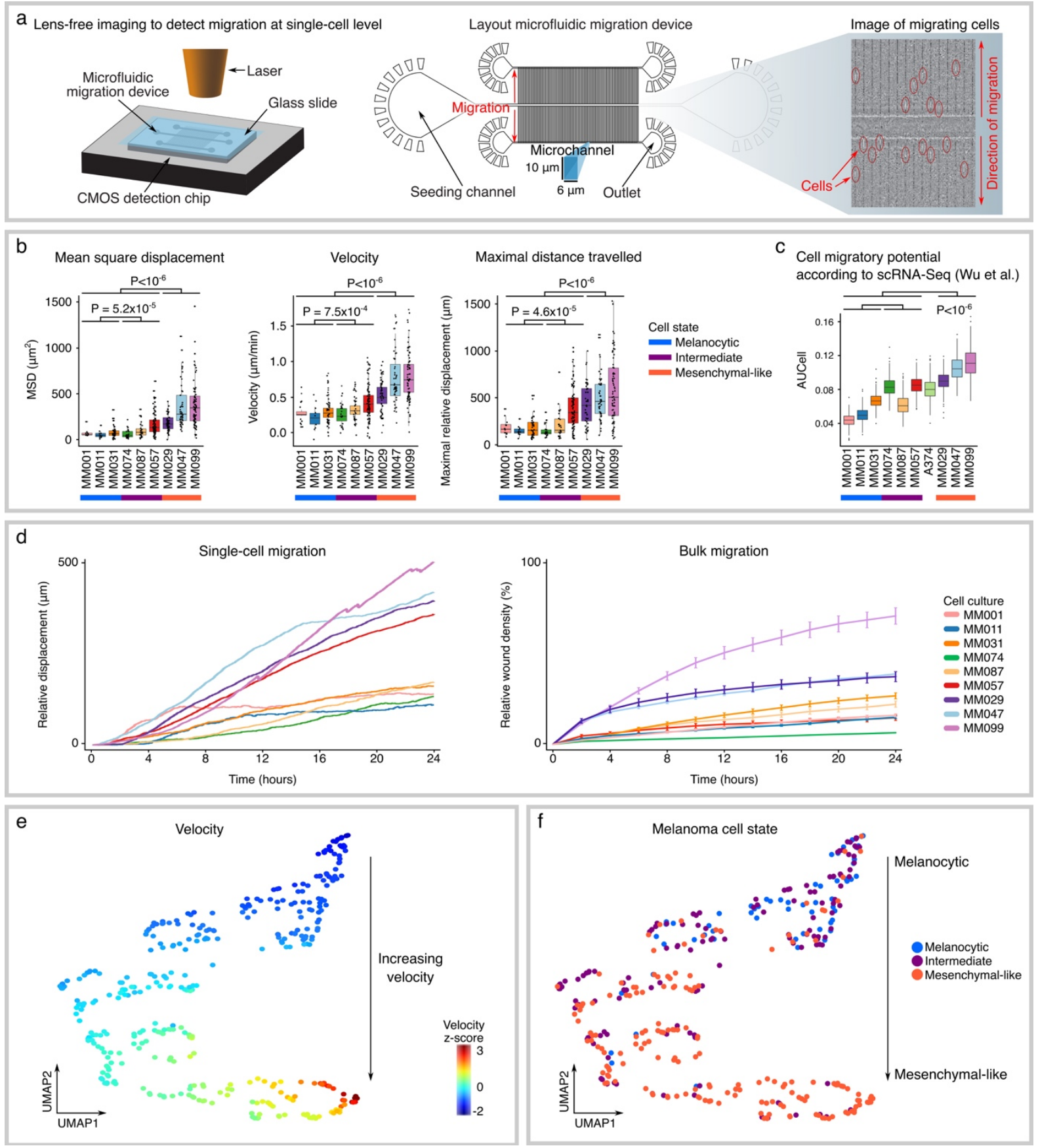
Figure 3

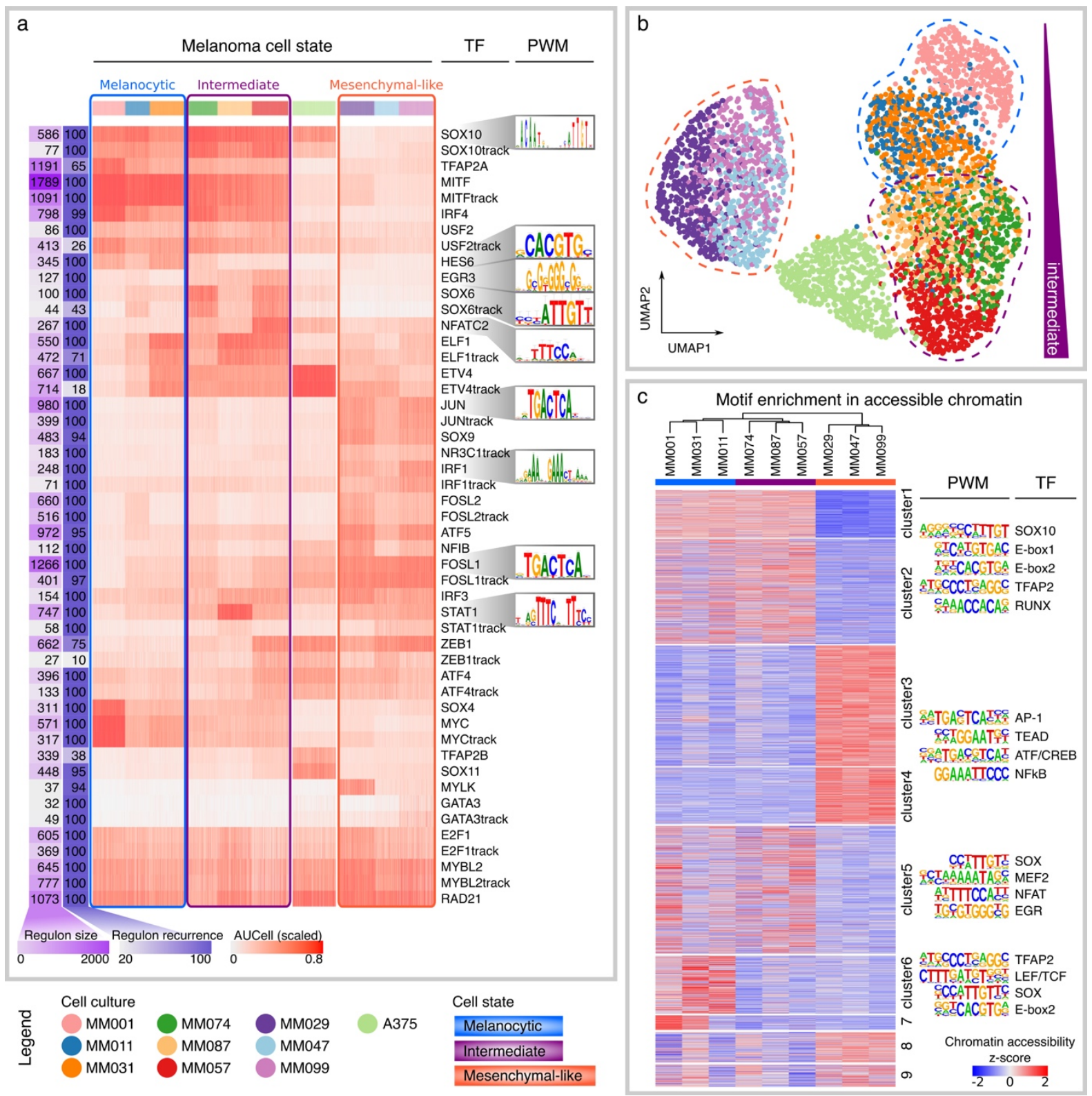


Figure 4

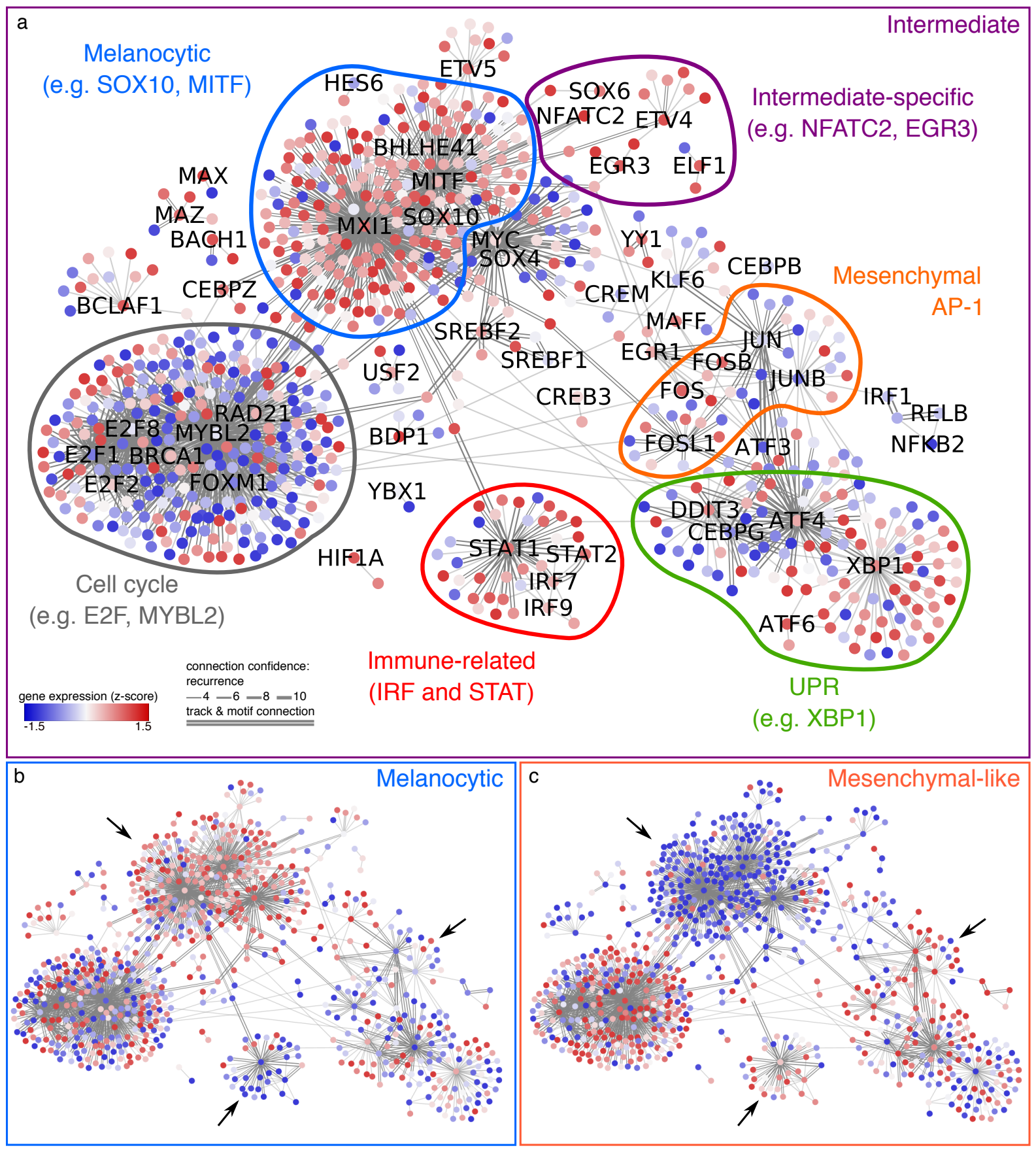


Figure 5
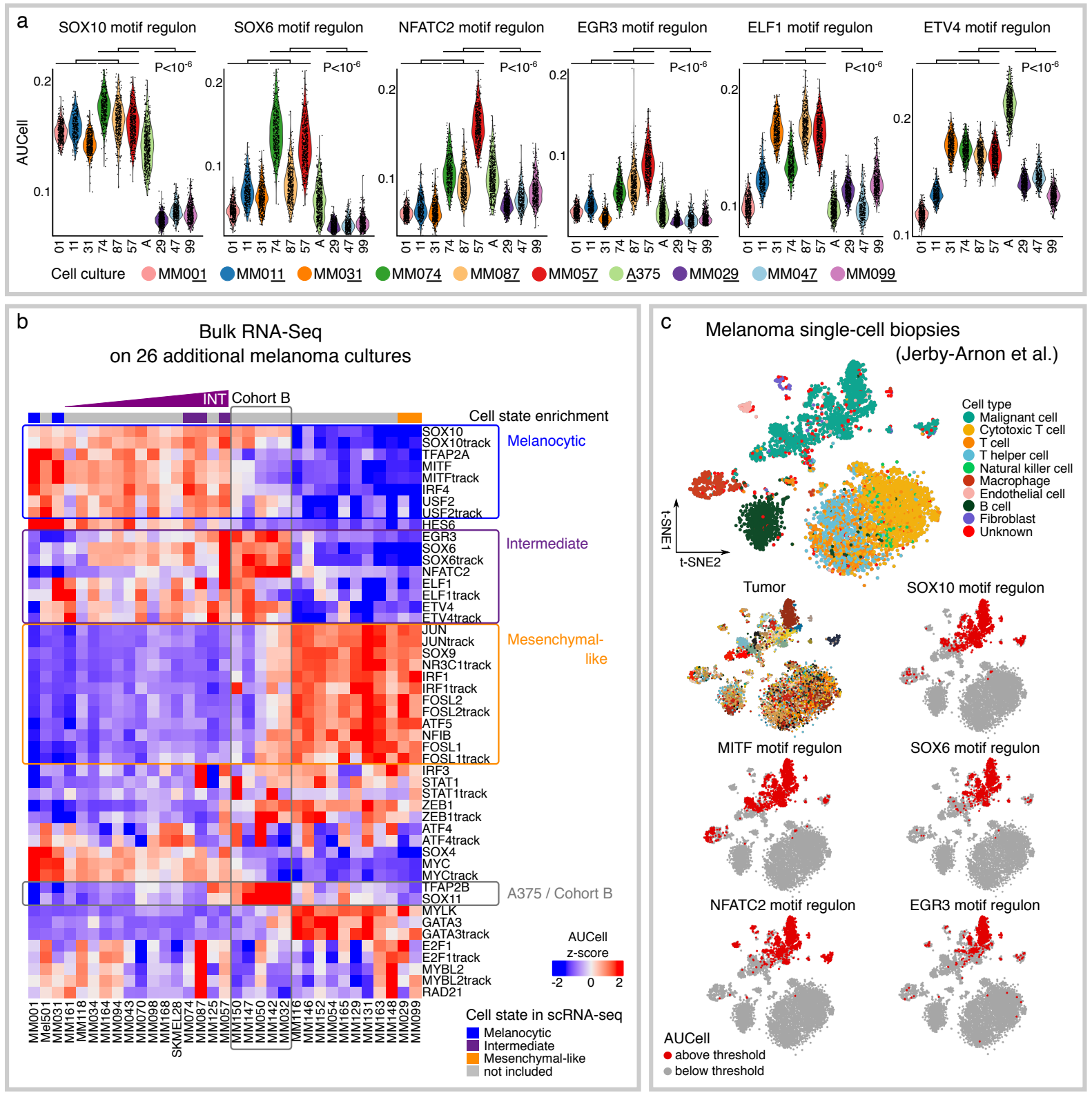


\section{Figure 6}

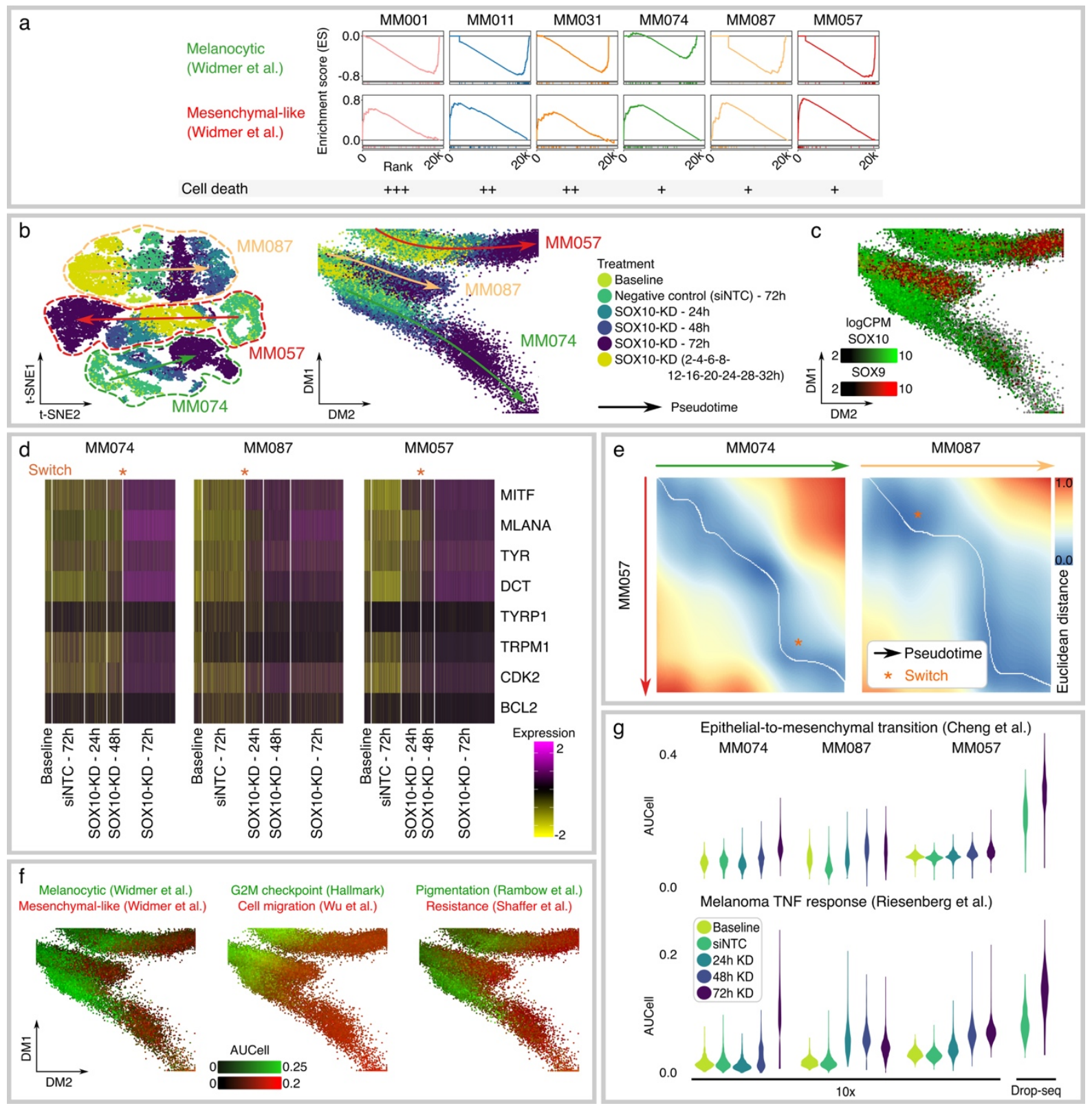


Figure 7
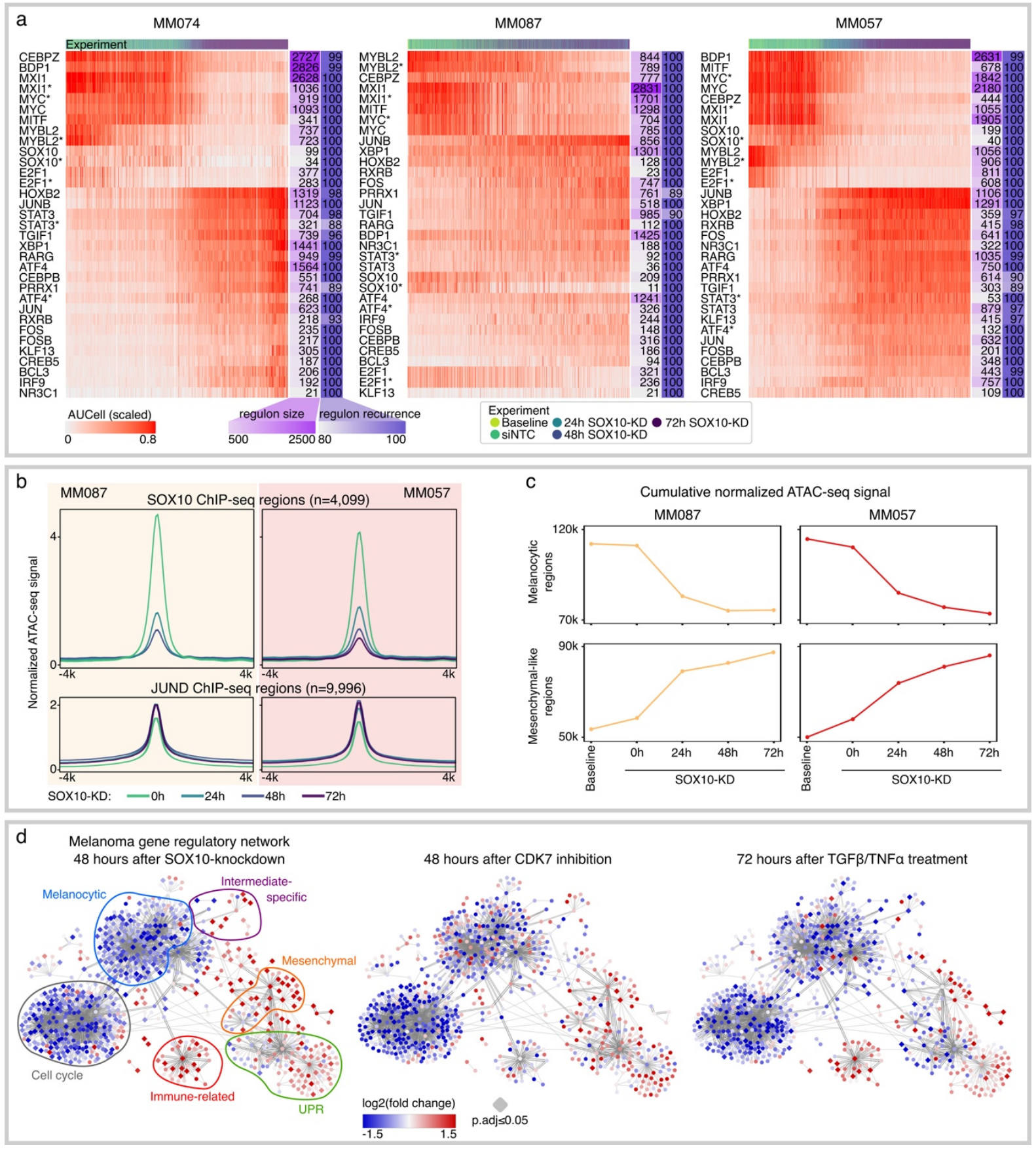